\title{
Interferon Status and Expression of P53 and gadd45g Genes in Leukaemogenic Felv Infection in Domestic Cats
}

\section{Alexander Tsybulsky ${ }^{*}$, Tatyana Tabakaeva ${ }^{1}$, Anna Klimovich ${ }^{2}$, Michail Shchelkanov ${ }^{1}$, Eduard Kostetsky ${ }^{1}$, Maksim Aliev ${ }^{1}$, Anton Degtyarenko ${ }^{3}$}

${ }^{1}$ Far Eastern Federal University, Russian Federation; ${ }^{2}$ Pacific Institute of Bioorganic Chemistry, FEBRAS, Russian Federation; ${ }^{3}$ Federal Scientific Center of Terrestrial Biodiversity of Eastern Asia, Far Eastern Branch of Russian Academy of Sciences, Russian Federation.

\begin{abstract}
Background. Feline Leukaemia Virus (FeLV) infection is a core aetiopathogenetic factor in the development of leukaemia in cats. The fundamental methods for such pathologies diagnostics, along with haematological analysis, include the replicative and expressive activity of FeLV evaluation in the infected organism cells, innate immune factors for antivirals evaluation, interferon system (IFN) in particular, molecular guard factors for cell genome integrity evaluation (p53, etc.). Methods. In domestic cats with a previously established diagnosis of «FeLV-infection and chronic leukaemia» $(n=10)$ and healthy control cats $(n=6)$, we examined the following: 1$)$ Haematological parameters of the blood; 2) Biochemical blood indicators of the physiological characteristics of the kidneys and liver, whose disorders are most critical in the progression of the leukaemic process; 3) Replicative and expressive activity of provirus FeLV in blood white cells (WBC); 4) Concentration of the IFNs and viral p27-FeLV-specific antigen in the blood plasma; 5) Expression of ifn $\alpha 6$, ifn $\alpha 7$, ifn $\beta 1$, if $n \gamma$ and ifn $\lambda 1$ genes in WBC; 6) Expression of $p 53$ and gadd45g genes in WBC. The concentration of the viral p27-FeLV antigen in the blood plasma was determined by ELISA. The total DNA and total RNA was extracted from the WBC. The cDNA was obtained using an mRNA matrix. Qualitative and quantitative analyses of FeLV in WBC as well as expression of genes of interest were conducted using real-time PCR (qPCR). Results. Cats with initial signs of leukaemic process activation in WBC showed the following: 1) enhanced FeLV replication activity, 2) reduced expression of the ifn $\alpha 6$, ifn $\alpha 7$, ifn $\lambda 1, p 53$, gadd $45 \mathrm{~g}$ genes and increased expression of the ifn $\beta 1$ gene in WBC, 3) decreased IFN classes $\alpha, \beta, \gamma$ concentration in blood plasma. Recent findings and conclusion. The diagnostic algorithm based on FeLV replicative activity analysis in animals with chronic leukaemia, as well as the state of the IFN system at phenotypic and genetic levels along with the analysis of the $p 53$ and gadd $45 \mathrm{~g}$ gene expression can be recommended for the examination of cats with FeLV-induced leukaemia to predict disease dynamics.
\end{abstract}

Keywords $\mid$ FeLV infection, leukaemia, haematological analysis, type I-II-III interferon, gene expression, gadd45g, p53.

Received | August 01, 2021; Accepted | December 09, 2021; Published | January 15, 2022

*Correspondence | Anguara Khatun, Far Eastern Federal University, Russian Federation; Email: avt_biotech@mail.ru

Citation | Tsybulsky A, Tabakaeva T, Klimovich A, Shchelkanov M, Kostetsky E, Aliev M, Degtyarenko A (2022). Interferon status and expression of $p 53$ and gadd45g genes in leukaemogenic felv infection in domestic cats. Adv. Anim. Vet. Sci. 10(3): 565-572.

DOI | http://dx.doi.org/10.17582/journal.aavs/2022/10.3.565.572

ISSN (Online) | 2307-8316

\section{INTRODUCTION}

$\mathrm{N}$ eoplastic diseases of the haematopoietic system (haemoblastosis) represent a large group of diseases of various aetiology and pathogenesis common in almost all

March 2022 | Volume 10 | Issue 3 | Page 565 mammalian species. With a number of haemoblastoses, the aetiopathogenic role of viruses has been proven, mainly from the families Retroviridae and Herpesviridae (Ciminale et al., 2014; Gazon et al., 2012; Shannon et al., 2017).

A specifc case of haemoblastosis is leukaemia, a malig- 
nant disease of blood and lymphatic tissues, which can be realised both in the form of systemic diseases of blood leukaemia and in the form of regional nodal tumour neoplasms - lymphoma and haematosarcoma. Leukaemia is common in almost all mammalian species and is particularly frequent in cats (including the domestic cat, Felis catus) (Lutz et al., 2009).

There is a high correlation between cat infection with FeLV retrovirus and the risk of leukaemia, which is that high that cat leukaemia can be seen as a FeLV-induced process (Cristo et al., 2019). It is obvious that the virus acts as an aetiopathogenetic factor in the initiation of haemato-oncological disease, and depending on the individual circumstances, it can be a factor in the promotion and progression of leukaemia.

For the treatment of diseases with such a manifesting viral component of aetiopathogenesis, promising antiviral therapies, in particular, recombinant IFN therapy and the development of effective induction methods for the synthesis of endogenous IFN, are suggested. In this context, this paper focuses on the evaluation of diagnostic algorithms to detect early signs of a chronic leukaemic process activation. This diagnostic algorithm allows for an assessment of the instability of the state of remission along with the risk of the transition of the clinical progression of leukaemia into a more aggressive stage. It is based on: (a) haematological and biochemical blood analysis, (b) molecular genetic study of FeLV replication, (c) INF concentration assessment classes $\alpha, \beta$ and $\gamma$ in blood plasma, (d) assessment of the expression levels of some target genes of the IFN system, (e) assessment of the expression levels of p53 and gadd $45 \mathrm{~g}$ (coding transcription factor TP53 and protein GADD45G (growth arrest and DNA-damage-inducible protein gamma), genes whose protein products are important components of the cell cycle control system.

\section{MATERIALS AND METHODS}

\section{Animals}

Domestic outbred cats $(\mathrm{n}=10)$, kept at standard conditions in the Vladivostok veterinary shelter, Russia. Previously, during veterinary examination, all animals were diagnosed with FeLV infection and leukaemia. The duration of the disease ranged from 6 months up to 2 years. The relevant indicators of healthy cats $(n=6)$ examined before performing planned sterilisation were used as control benchmarks.

\section{ETHICAL STANDARDS}

All manipulations with animals were carried out in accordance with the regulations approved for veterinary clinics in Vladivostok.

\section{Biological MATERIAL}

Peripheral blood of animals taken at a volume of $2 \mathrm{~mL}$ from the lateral vein of the forelimb into vacuum tubes containing EDTA potassium salt as anticoagulant.

Haematological panel: was performed using the Cell Dyn 3700 automatic haematology analyser (Abbott, USA). Of the general spectrum of haematological data, the following parameters were used: 1) WBC absolute content in G/L, 2) Neutrophils stab (Neu st), Neutrophils segmented (Neu se), Lymphocytes (Lymph), and Monocytes (Mon) relative content (in \%), 3) erythrocyte sedimentation rate (ESR) in $\mathrm{mm} /$ hour.

The determination of the WBC blast forms in blood was carried out through analysis of a microscopic blood smear. According to the haematological panel results, all animals were divided into two groups: (1) cats in remission of chronic leukaemia and (2) cats with initial signs of leukaemic process activation (increased WBC count).

Biochemical blood tests: were performed using the URIT-800 VET (URIT Medical Electronic Co., Ltd., China) biochemical analyser. The following parameters were tested: creatinine (in $\mu \mathrm{mol} / \mathrm{L}$ ), urea (in $\mathrm{mmol} / \mathrm{L}$ ), residual nitrogen (in $\mathrm{mmol} / \mathrm{L}$ ), glucose (in $\mathrm{mmol} / \mathrm{L}$ ), total cholesterol (mmol/L), activity of amylase and AST and ALT transaminases (in $\mathrm{U} / \mathrm{ml}$ ), and total blood protein (in g/L).

FeLV p27 virus-specific antigen testing: in blood plasma was conducted with the ELISA method, using the FeLV p27 antigen ELISA diagnostic kit (Demeditec, Germany) under the manufacturer's recommendations.

\section{Molecular genetic examination of FeLV provirus in the} cat WBC: The extraction of total nuclear DNA from WBC was carried out using a set of «DNA sorb B» («Amplisens», Russia) following the manufacturer's recommendations. Analysis of the concentration and quality of isolated DNA was carried out using the spectrophotometer NanoDrop 2000C («Thermo Scientific», USA). The FeLV provirus was detected with an application of the FRT PCR with the Thermocycler Real-Time CFX96 Touch (BioRAD, USA) and the LEIKIS diagnostic kit (Amplisens, Russia), following the manufacturer's recommendations.

Along with a qualitative assessment of the presence of provirus FelV in WBC, it was quantified. To this end, a positive reference sample with a known viral DNA concentration was titrated (dilution coefficient $=\log 2$ ), and a calibration curve was generated based on $\mathrm{Ct}$ indicators. The data were approximated for an $\mathrm{R}^{2}$ of 0.95 . The calculated values obtained were expressed in relative units 
of the provirus concentration of FeLV per $1 \mathrm{~mL}$ DNA sample.

Analysis of target gene expression: Extraction of the total RNA from the cat WBC was performed with an application of the ExtractRNA reagent (Eurogen, Russia), according to the manufacturer's recommendations. The concentration and quality of the isolated RNA were analysed with a NanoDrop 2000C spectrophotometer (Thermo Scientific, USA). Reverse transcription needed to obtain a single-stranded cDNA on the mRNA matrix was performed with an application of the MMLV RT reagent kit (Eurogen, Russia) with the Oligo(dT) primer. All procedures were performed according to the manufacturer's recommendations.

The expression of the following feline genes was analysed: (a) type I IFN (ifn $\alpha 6$, ifn $\alpha 7$ and ifn $\beta 1$ ), (b) type II IFN

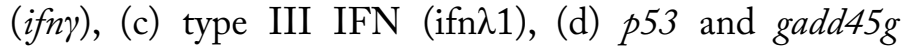
genes of the cell cycle control, (e) gapdh (glyceraldehyde 3-phosphate dehydrogenase).

Primer-BLAST NCBI and SnapGene 4.1.8 were used to design the primers. The following pairs of forward $(\mathrm{F})$ and reverse $(\mathrm{R})$ gene-specific primers were used (Table 1$)$.

Gene expression analysis was carried out using the qPCR mix HS SYBR («Eurogen», Russia) by qPCR, applying the thermocycler CFX96 («BIORAD», USA). The gapdh gene was used as a normaliser gene. Samples of cDNA derived from mRNAs of practically healthy cats were used as control. The relative differences in the expression of each gene in Group 2 compared to Group 1 were obtained by calculating the multiplicity of expression variation (fold) according to a previously described method (Livak and Schmittgen, 2001).

Quantitative analysis of IFN classes $\alpha, \beta$ and $\boldsymbol{\gamma}$ in blood plasma of cats was performed by ELISA, using Cat IFN $\alpha$ ELISA KIT, Cat IFN $\beta$ ELISA KIT and Cat IFN $\gamma$ ELISA KIT (Bioassay Technology Lab., China) according to the manufacturer's recommendations. Analysis results were detected on the microplate photometer ELx808iu (BioTek, USA) at a wavelength of $450 \mathrm{~nm}$; IFN concentrations were expressed in $\mu \mathrm{g} / \mathrm{mL}$ of blood plasma.

Statistical processing of the data was carried out using the SPSS 11.0 statistical analysis software with the determination of the Fisher-Student confidence criterion. Differences at $p<0.05$ were considered significant.

The results were expressed as arithmetic mean and standard deviation $(\mathrm{M} \pm \mathrm{SEM})$. The Fisher-Student confidence criterion was used to evaluate the significance of results.
Differences were considered statistically significant at $\mathrm{p}<0.05$.

\section{RESULTS}

Haematological analysis of the peripheral blood of cats: All examined animals with a previously established diagnosis of FeLV infection and chronic leukaemia did not demonstrate any statistically significant changes in parameters of the red blood as well as signs of thrombocytopenia that are typical for progressive FeLV infection (Biezus et al., 2019). Haematocrit values ranged from $30-40 \%$.

The general condition of all cats with leukaemia: was assessed by veterinarians as relatively satisfactory. All animals were in remission of the main disease (i.e., chronic leukaemia), which was confirmed by the analysis of leukogram and blood biochemistry. There were no significant changes in parameters indicating multiple organ failure (typical of leukaemia onset and, especially, blast crisis), such as urea, creatinine, and blood plasma residual nitrogen concentrations. Likewise, there was no evidence of deviation from the physiological standards of the glucose content, total cholesterol, and total blood protein. However, it should be noted that blood urea and creatinine were at the upper normal levels for healthy animals (Table 1). These findings suggest a likely over-compensation in these animals and serve as an indication for more in-depth examination and therapeutic interventions to secure and prolong the remission stage. However, analysing haematological parameters, we found animals with significantly different absolute WBC levels. Based on this parameter, two groups of animals were formed, each containing five cats: (1) cats with WBCs within the range of 4.6-10 G/L (on average, $7.0 \pm 1.9 \mathrm{G} / \mathrm{L}$ ) and (2) cats with WBCs within the range of $12.6-20.2 \mathrm{G} / \mathrm{L}(15.8 \pm 2.8 \mathrm{G} / \mathrm{L})$. The WBC counts in healthy control cats were within $7.5 \pm 2.7 \mathrm{G} / \mathrm{L}$ (Table 2).

No blast forms of WBC in blood smears in any of the animals were found. However, Group 2 cats had leucocytosis at the upper levels of the WBC count in control animals, even noticeably higher than the reference. These signs of leucocytosis in this group of cats can be estimated from two positions: (1) as a consequence of some chronic leukaemic process activation or (2) as a sign of the system-wide inflammatory process development.

Typical biochemical inflammation markers, such as transaminase activity ALT and AST, were characterised: (1) in Group 1, the activity of ALT and AST was $75.2 \pm$ 10.5 and $58 \pm 11 \mathrm{U} / \mathrm{mL}$, respectively, (2) in Group 2, the activity of ALT and AST was $66.8 \pm 11.7$ and $54 \pm 10 \mathrm{U} /$ $\mathrm{mL}$, respectively (with the results in control animals of 
Table 1: Primer sequences specific for feline target genes

\begin{tabular}{|c|c|c|}
\hline Feline gene & Forvard primer $\left(5^{\prime}-3^{\prime}\right)$ & Reverse primer $\left(5^{\prime}-3^{\prime}\right)$ \\
\hline ifna6 & GGCGTCCTCGTCTGCTGCTT & GGATGGAGTCCTCGTTCGTGAG \\
\hline ifna7 & GGACATTCATCCCGAGGAC & GACATGGCAGGACAGGTGGA \\
\hline ifn $\beta 1$ & AATCACAGCGGTTCCAGAAG & CTTCAGGTACCGCACAATCC \\
\hline ifn $\gamma$ & ATCCAGATGTAGCAGATGGTGGG & TCCATGCTCCTTTGAATGCG \\
\hline ifn $\lambda 1$ & GGGACCTGAGACAGCTACAG & TCCTCAGACACACAGGTCTC \\
\hline gadd $45 \mathrm{~g}$ & TGCAGATCCACTTTACGCTGA & GAGGATGCAGTGTAGGTCTCC \\
\hline p53 & TGACAGAGGTCGTGAGGCG & AGGGTGATGATGGTGATGATGG \\
\hline gapdh & TGACCCCTTCATTGACCTCA & TTCACGCCCATCACAAACAT \\
\hline
\end{tabular}

Table 2: Biochemical parameters of blood in cats with FeLV-induced leukemia $(\mathrm{M} \pm \mathrm{SEM})$.

\begin{tabular}{|llllllll} 
Group & $\begin{array}{l}\text { Number of } \\
\text { cats }\end{array}$ & $\begin{array}{l}\text { Urea } \\
\mathbf{m m o l} / \mathbf{L}\end{array}$ & $\begin{array}{l}\text { Residual } \\
\text { nitrogen } \\
\mathbf{m m o l} / \mathbf{L}\end{array}$ & $\begin{array}{l}\text { Creatinine } \\
\mathbf{m k m o l} / \mathbf{L}\end{array}$ & $\begin{array}{l}\text { Total protein } \\
\mathbf{g} / \mathbf{L}\end{array}$ & $\begin{array}{l}\text { Glucose } \\
\mathbf{m m o l} / \mathbf{L}\end{array}$ & $\begin{array}{l}\text { Total } \\
\text { cholesterol } \\
\text { mmol/L }\end{array}$ \\
\hline FeLV-infected cats & 10 & $7,4 \pm 2,8$ & $17,8 \pm 3,5$ & $96 \pm 21$ & $60,2 \pm 1,1$ & $3,2 \pm 0,5$ & $3,8 \pm 1,1$ \\
\hline Control $^{*}$ & 6 & $6.4 \pm 2.1$ & $15.3 \pm 2.6$ & $68 \pm 11$ & $59.6 \pm 2.8$ & $3.8 \pm 0.4$ & $3.5 \pm 1.3$ \\
\hline
\end{tabular}

* healthy cats examined before performing planned sterilization.

Table 3: Haematological parameters in cats with leukaemia $(\mathrm{M} \pm \mathrm{SEM})$.

\begin{tabular}{llllllll} 
Group & $\begin{array}{l}\text { Number } \\
\text { of cats }\end{array}$ & $\begin{array}{l}\text { WBC } \\
\text { G/L }\end{array}$ & $\begin{array}{l}\text { Neu st } \\
\mathbf{\%}\end{array}$ & $\begin{array}{l}\text { Neu se } \\
\mathbf{\%}\end{array}$ & $\begin{array}{l}\text { Lymph } \\
\mathbf{\%}\end{array}$ & $\begin{array}{l}\text { Mon } \\
\mathbf{\%}\end{array}$ & $\begin{array}{l}\text { ESR } \\
\mathbf{m m} / \mathbf{h}\end{array}$ \\
\hline 1 & 5 & $7,0 \pm 1,9$ & $0.6 \pm 0.4$ & $65 \pm 5^{*}$ & $34 \pm 6$ & $0,8 \pm 0,7$ & $8,7 \pm 6,1$ \\
2 & 5 & $15,8 \pm 2,8^{*}$ & $2.0 \pm 0.6$ & $71 \pm 1^{*}$ & $24 \pm 3^{*}$ & $2,0 \pm 0,9$ & $5,6 \pm 2,7$ \\
Control & 6 & $7.5 \pm 2,7$ & $1.1 \pm 0.5$ & $44 \pm 3$ & $42 \pm 6$ & $1.8 \pm 0.6$ & $6.2 \pm 1.4$ \\
Notes: Group 1 - cats in stable remission of leukemia, Group 2 - cats with initial signs of leukemia activation, Control - healthy cats. \\
*- The differences versus the control group are statistically significant $(\mathrm{p}<0.05)$.
\end{tabular}

$37.4 \pm 9.2$ and $14.8 \pm 6.3 \mathrm{U} / \mathrm{ml}$, respectively). There were no statistically significant differences between the experimental groups ( $p>0.05$ ), but there was a noticeable increase in AST and ALT activity in both experimental groups as compared to the control group ( $p<0.05$ ). These transaminase activity levels are predictably unfavourable for both experimental groups of cats with Feline Leukaemia.

Analysis of another inflammation marker, ESR (Table 2), also found no significant differences between the two examined groups, strongly suggesting that leucocytosis in Group 2 cats was a sign of an onset of leukaemia as the underlying disease.

In this regard, the criteria for the early detection of the leukaemic process activation signs and the determination of indications for adequate measures to extend the remission stage are therefore of utmost importance. We used a combination of three blocks of diagnostic tests as such prognostic criteria: (1) assessment of FeLV replication activity, (2) assessment of the IFN system and (3) assessment of the levels of p53 and gadd $45 \mathrm{~g}$ gene expression.
FeLV replication activity: When PCR-tested for FeLV in WBC, all Group 2 cats were positive for FeLV (100\%), whereas in Group 1, only one cat out of five examined was identified with FeLV. When these data were approximated concerning the concentration function $y=f(C t)$ for a positive provirus standard sample (with a DNA concentration of $21 \mathrm{ug} / \mathrm{mL}$ ), a rather low concentration of provirus DNA in the DNA samples of the examined cats was found (range $0-0.3 \mu \mathrm{g} / \mathrm{mL}$ ). However, one cat in Group 2 had a significantly increased provirus DNA concentration $(17.76 \mu \mathrm{g} / \mathrm{mL})$, indicating a high replication activity of the virus in the $\mathrm{WBC}$ of this animal.

At the same time, the FeLV expression activity in the WBC of all animals was low: only two cats from Group 2 and one cat from Group 1 were weak seropositive in the content of p27-FeLV-specific antigen in blood plasma. The remaining seven cats were seronegative to this FeLV antigen.

Levels of IFN classes $\alpha, \beta$ and $\gamma$ in blood: Analysis of feline-specific IFN concentrations in blood plasma showed no statistically significant difference between the two 
groups of leukaemia patients indicated below (Figure 1).

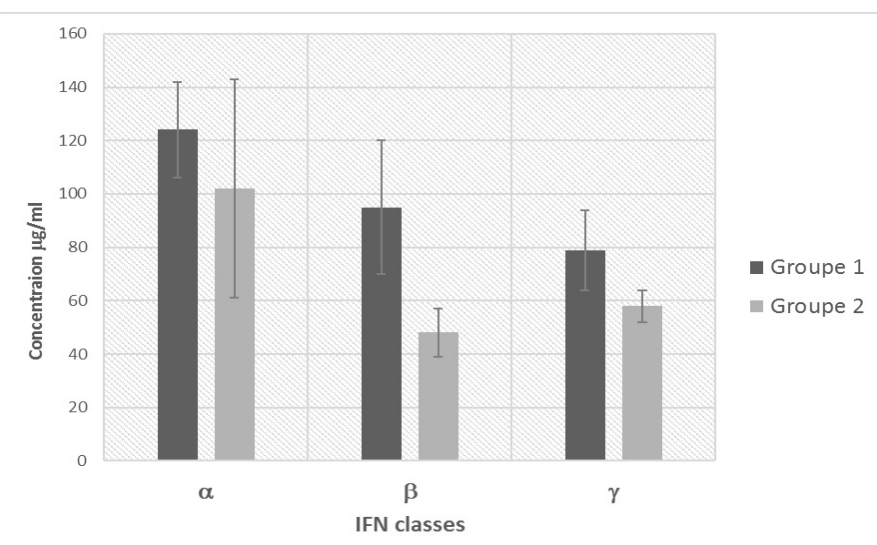

Figure 1: Plasma IFN concentrations in group 1 and group 2 cats with leukaemogenic FeLV-infection.

Group 1 - cats with aleukaemic stage of leukaemia, group 2 - subleukaemic stage of leukaemia.

However, there was a rather pronounced general trend: the levels of all three IFN classes in Group 2 were lower than those in Group 1 . This difference was especially significant concerning IFN $\beta$, which has both noticeable antitumor and antiviral activity. We consider this feature of interferon status in cats with FeLV-induced leukaemia to be highly important for the development of new protocols for the treatment of this pathology.

In this regard, we also deemed it interesting to determine the parameters of the IFN system gene expression. This is a large family of structural, regulatory and IFN-inducible genes. Of these, we selected ifn $\alpha 6$, ifn $\alpha 7$, ifn $\beta 1$, ifn $\gamma$ and ifn $\lambda 1$ genes for analysis. These specific IFN genes were selected for the expression analysis because of the following: (1) our bioinformatics analysis revealed antimitotic, apoptosis-regulating and antitumor activity of the corresponding protein products, (2) ifn $\alpha$, ifn $\beta 1$, ifn $\gamma$ and ifn $\lambda 1$ genes are located fairly far away from each other on the phylogenetic tree (we assume that such a distance can be associated with a significant difference in the spectrum of biological activity of these genes). Analysis of the relative expression of these genes in the peripheral blood leukocytes of cats gave the following results ( Figure 2).

The WBC's of Group 2 cats showed significantly lower expression levels of ifn $\alpha 6$, ifn $\alpha 7$ and ifn $\lambda 1$ as well as of important factors of cellular genome health, such as p53 and gadd $45 \mathrm{~g}$. The expression level of ifn $\gamma$ was almost the same, whereas that of the ifn $\beta 1$ gene was by about $50 \%$ higher. These data do not fully correspond to the results of ELISA concerning the IFN $\alpha$, IFN $\beta$ and IFN $\gamma$ concentrations in the cat blood plasma due to some obvious reasons: (a) peripheral blood leukocytes are not the main producers of IFN $\beta$, and (b) IFN $\alpha$, IFN $\gamma$ and other IFNs are mainly synthesised by immunocompetent cells in the peripheral organs of the immune system. This fact was repeatedly counteracted in our experiments, where on the periphery in situ (in particular in the area of local inflammation), the concentration of cytokines was significantly higher than that in the system circulation.

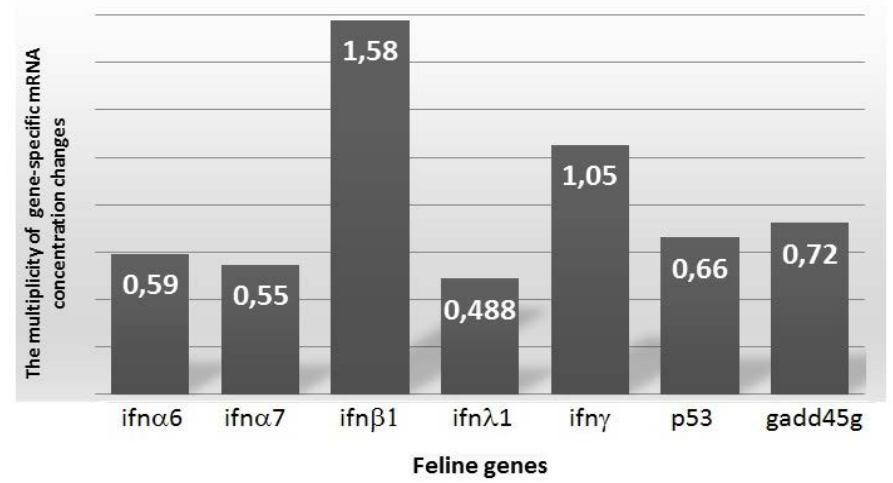

Figure 2: Differences in expression of interferon, p53 and gadd $45 \mathrm{~g}$ genes in blood leukocytes from group 2 cats relative to group 1 .

Relative expression levels of target genes normalized by the gapdh. The values presented are quotients of the expression values in group 2 by the expression values in group 1 (Fold2 / Fold1).

In general, by evaluating the data presented in Figure 2, we can conclude that the expression reduction of a given group of genes is a marker of the negative scenario of the FeLV-induced leukaemic process. Such a decrease in the expression levels of these genes, which are important factors of antiviral and antitumor protection, creates conditions for a higher replication activity of the virus and an increased leukemogenic and tumorigenic potential of the FeLV infection.

\section{DISCUSSION}

Significant differences were found in the parameters of viral activity, IFN status and expression of cell cycle control genes between two groups of FeLV-infected cats. Both groups did not show clinical signs of leukaemia activation and the transition of the disease into the blast crisis phase, but had significant differences in leucocytosis indicators (aleukemic and subleukaemic forms). The identified differences can serve as criteria for the prediction and early detection of leukaemia activation and, possibly, as markers for monitoring the effectiveness of preventive and curative interventions.

The essence of the diagnostic algorithm we propose is the use of a combination of diagnostic tests aimed at identifying the interrelationship of four parameters: «Haematological markers of leukemic activity - Replicative activity 
of FeLV in WBC - Expressive activity of some genes of the IFN system, along with p53 and gadd $45 \mathrm{~g}$ genes». This combination of tests achieves an assessment of the peripheral blood pattern, the state of the IFN system and the characteristics of the replicative activity of the FeLV, which may play a role as the initiator of the leukaemic process, and its promoter and driver during the progression stage of the disease. Such a pronounced role of the FeLV to the pathogenesis of feline leukaemia is a direct indication of the need to monitor FeLV replication activity in sick animals.

It should be mentioned that the expression of the virus activity at certain stages of FeLV infection may be completely non-existent (Hartmann, 2012), as also shown in our study. Only two cats from Group 2 and one cat from Group 1 were slightly seropositive for the content of $\mathrm{p} 27-\mathrm{FeLV}$-specific antigen in the blood plasma. The remaining seven cats with chronic leukaemia were seronegative for this viral antigen, irrespective of the haematological signs of the leukaemic process activation. Therefore, assessment of the content of virus-specific antigens in the blood of animals with leukaemia has demonstrated a rather low-resolution capacity. Probably, the assessment of seroconversion of the blood in sick animals by anti-FeLV antibodies can be more informative. However, in turn, the relevance of this type of testing also has its limitations since seroconversion can be the result of contact with the viral material where the induction of the leukaemic process does not occur.

In a number of cases, the replicative activity of the FeLV virus in the blood cells of animals with leukaemia may be non-existent. However, such cases are not a part of any regular pattern. In particular, in our study, all cats in Group 2 were positive for FeLV. The detection of the replicative activity of the virus is a major alarm that requires a decision to be taken to implement measures aimed at preventing the disruption of the state of remission in patients with leukaemia.

The status of the IFN system in any form of virus-induced pathologies, among others, including virus-induced cancer pathology, has an obvious formativeness. The IFN status research provides the following information: (1) it characterises the state of one of the most important antiviral systems of the innate immunity of the animal body, (2) it assesses the intensity of the virus immunosuppressive effect and (3) it characterises the dynamics of the disease and the effectiveness of the therapeutic and preventive measures. Our research findings indicate that when the leukaemic process is onset in cats, the IFN system is inhibited both at the phenotypic level (which is manifested in a decline in the concentration of IFN $\alpha, \beta$ and $\gamma$ classes in the blood plasma) and at the level of expression of a num- ber of structural IFN genes $(a 6, \alpha 7, \lambda 1)$. It is highly probable that effective antiviral and antitumor protection of the animal body requires not just an activation but rather an over-expression of these IFN genes. In this regard, it can be noted, for example, that effective treatment of hepatitis $\mathrm{C}$ and a number of other diseases requires long-term administration of high doses of IFN (Feld and Hoofnagle, 2005; Rong and Perelson, 2010).

The importance of the expression analysis of $\mathrm{p} 53$ and gad$\mathrm{d} 45 \mathrm{~g}$ genes stems from the fact that the protein products of these genes have a high biological activity spectrum and are key factors of genetic homeostasis and cell cycle control. In the blood leukocytes of cats in Group 2, we found a significantly reduced expression of these genes.

The most obvious direct and inverse relationships found in this study during the comparison of the diagnostic parameters typical for animals in Groups 1 and 2 are shown in Figure 3.

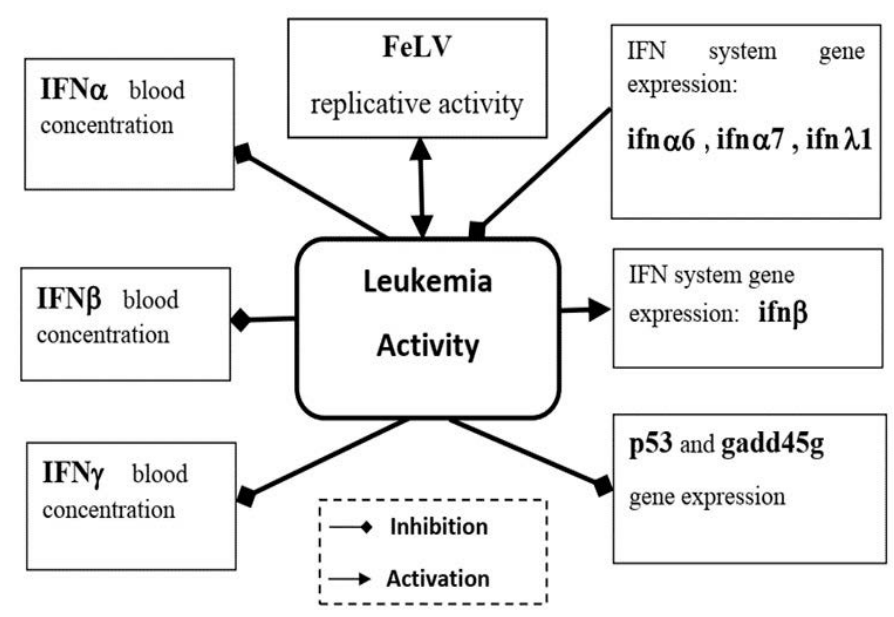

Figure 3: Dependence of the leukaemic process activity and parameters that characterise the replicative activity of FeLV, the concentration of IFN in the blood and the expression of certain genes of the IFN system and the p53 and gadd $45 \mathrm{~g}$ genes.

Overall, looking at the data presented in Figure 3, it can be concluded that the declining expression of this group of genes is a marker of a negative scenario for the course of FeLV-induced leukaemia. Such a decline in the expression of these factors of antiviral and antitumor protection creates suitable conditions for a higher replication activity of the virus and an increasing leukaemogenic and tumorigenic potential of the FeLV infection.

\section{CONCLUSIONS}

The increased level of FeLV replication in WBC of cats with initial signs of leukaemia activation suggests the im- 
portance of analysing this indicator to assess the risk of a possible remission failure.

However, we have not been able to identify a clear quantitative relationship that characterises the replication activity of the virus and the parameters of blood leucocytosis. Thus, this test has relative predictive capabilities. At the same time, the combination of (a) haematological analysis, (b) molecular genetic analysis of the replication activity of the virus and (c) estimation of the status of antiviral and antitumour resistance factors (some IFN genes and cell cycle control genes) is a fairly informative diagnostic approach. We believe that the assessment of the viral activity and the state of the IFN system, together with the assessment of the factors P53 and GADD45g, can provide the possibility of an earlier detection of the risks of activating the leukaemia process in animals with FeLV-induced leukaemia.

The analysis of these parameters by molecular methods at the level of gene expression is naturally of less diagnostic value than the estimation of the concentration and activity of protein products. However, such analysis is much cheaper and is available in any laboratory equipped with a qPCR analysis apparatus.

Naturally, the IFN I-II-III spectrum we studied does not limit the IFN system evaluation. Particularly relevant may be the analysis, for example, of feline-specific IFN $\omega$, the recombinant preparation of which is almost exclusively used for the treatment of many diseases of viral genesis in animals (and not only in cats). Nevertheless, we can summarise that the developed diagnostic algorithm combining (a) genetic analysis of FeLV replication activity, (b) phenotypic assessment of the state of IFN systems, (c) analysis of the expression levels of some genes of IFN system, as well as p53 and gadd $45 \mathrm{~g}$ genes, has a high diagnostic resolution. This algorithm may be useful for assessing the condition of cats with leukaemia to predict the development of the disease, as well as for developing new protocols for antiviral and antitumor therapy.

\section{ETHICS APPROVAL AND CONSENT TO PARTICIPATE}

This research was approved by ethical standards governing the work of veterinary clinics in Vladivostok. There was a study of samples collected with full informed consent from owners who agreed to the use of samples for research purposes.

\section{ACKNOWLEDGEMENTS}

The authors thank the veterinary practices and cat breeders who helped in the acquisition of samples used in this study.

\section{CONFLICT OF INTERESTS}

No competing financial interests exist.

FUNDING

This research was supported by the RFBR grant (Agreement No. 18-29-09060/18).

\section{AUTHORS' CONTRIBUTIONS}

Each author made substantial contributions to the conception and design of the work, the acquisition and the analysis of blood and DNA/RNA samples. TA performed the final analysis of all results and prepared the final version of this original article. All authors read and approved the final manuscript.

\section{REFERENCES}

Biezus G, Ferian PE, da Silva Pereira LHH, Withoeft JA, Antunes MM, Xavier MGN, Casagrande RA (2019). Clinical and haematological disorders in cats with natural and progressive infection by Feline Leukemia Virus. Acta Sci. Vet. 47: 1-9, 2019 https://doi.org/10.22456/16799216.90027

Ciminale V, Rende F, Bertazzoni U, and Romanelli MG (2014). HTLV-1 and HTLV-2: highly similar viruses with distinct oncogenic properties. Frontiers in Microbiology 5:1-9 https://doi.org/10.3389/fmicb.2014.00398 4

Cristo TG, Biezus G, Noronha LF, Gaspar T, Dal Pont TP, Withoeft JA, Furlan LV., Costa LS.,Traverso SD, Casagrande RA (2019). Feline Leukaemia Virus Associated with Leukaemia in Cats in Santa Catarina, Brazil. J. Comp. Pathol. 170: 10-21. https://doi.org/10.1016/j. jсpa.2019.05.002

Feld JJ, Hoofnagle JH (2005). Mechanism of action of interferon and ribavirin in treatment of hepatitis C. Nature 436: $967-$ 72. https://doi.org/10.1038/nature04082

Gazon, H, Lemasson, I, Polakowski N, Césaire R, Matsuoka M, Barbeau B, ... Peloponese, JM (2012). Human T-cell leukemia virus type 1 (HTLV-1) bZIP factor requires cellular transcription factor JunD to upregulate HTLV-1 antisense transcription from the 3 long terminal repeat $\mathrm{J}$. Virol. 86: 9070-9078. https://doi.org/10.1128/JVI.0066112

Hartmann K (2012). Clinical Aspects of Feline Retroviruses: A Review. Viruses. 4: 2684-2710. https://doi.org/10.3390/ v4112684

Livak KJ, Schmittgen TD (2001). Analysis of Relative Gene Expression Data Using RealTime Quantitative PCR and the 22DDCT Method. Methods. 25: 402-408. https://doi. org/10.1006/meth.2001.1262

Lutz H, Addie D, Belák S, Boucraut-Baralon C., Egberink H, Frymus T, Marsilio F (2009). Feline leukaemia ABCD guidelines on prevention and management (Review). J. Feline Med. Surg. 11: 565-574. https://doi.org/10.1016/j. jfms.2009.05.005

Rong L, Perelson AS (2010). Treatment of hepatitis C virus 
infection with interferon and small molecule direct antivirals: viral kinetics and modeling. Crit.Rev. Immunol. 30: 131-148. https://doi.org/10.1615/CritRevImmunol. v30.i2.30
Shannon-Lowe C, Rickinson AB, Bell A (2017). Epstein-Barr virus-associated lymphomas. PHILOS. T. R. SOC. B. 372:1-15. https://doi.org/10.1098/rstb.2016.0271 